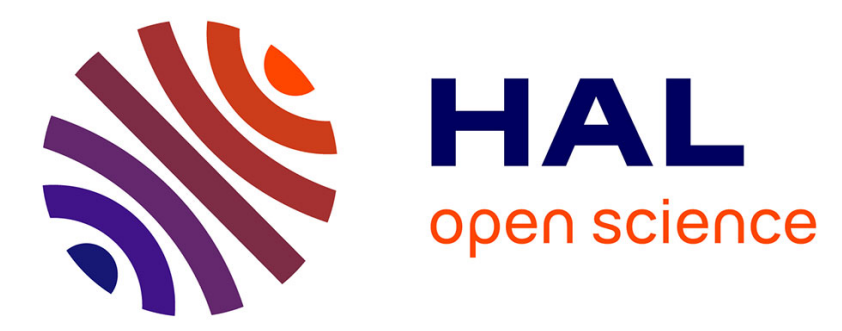

\title{
L'échec de la certification forestière. Le cas de la certification FSC examiné au travers de la sociologie de la traduction
}

Louise Briec, Matthieu Mandard

\section{- To cite this version:}

Louise Briec, Matthieu Mandard. L'échec de la certification forestière. Le cas de la certification FSC examiné au travers de la sociologie de la traduction. Mondes en Développement, 2016, 1 (173), pp.63-78. 10.3917/med.173.0063 . halshs-01298087

\section{HAL Id: halshs-01298087 \\ https://shs.hal.science/halshs-01298087}

Submitted on 15 Apr 2021

HAL is a multi-disciplinary open access archive for the deposit and dissemination of scientific research documents, whether they are published or not. The documents may come from teaching and research institutions in France or abroad, or from public or private research centers.
L'archive ouverte pluridisciplinaire HAL, est destinée au dépôt et à la diffusion de documents scientifiques de niveau recherche, publiés ou non, émanant des établissements d'enseignement et de recherche français ou étrangers, des laboratoires publics ou privés. 


\title{
L'ÉCHEC DE LA CERTIFICATION FORESTIÈRE
}

Le cas de la certification FSC à la lumière de la sociologie de la traduction

\author{
Louise Briec \\ Université Lille 1 \\ Centre Lillois d'Études et de Recherches Sociologiques et Économiques \\ (CLERSÉ - UMR CNRS 8019) \\ louise.briec@ed.univ-lille1.fr
}

\author{
Matthieu Mandard \\ Université Rennes 1 \\ Centre de Recherche en Économie et Management \\ (CREM -UMR CNRS 6211) \\ matthieu.mandard@univ-rennes1.fr
}




\title{
L'ÉCHEC DE LA CERTIFICATION FORESTIÈRE
}

\section{Le cas de la certification FSC à la lumière de la sociologie de la traduction}

Résumé : Initiée dans les années 1990, la certification forestière a pour objectif de promouvoir une gestion forestière durable. Malgré les efforts consacrés à sa mise en œuvre, elle ne rencontre cependant pas le succès escompté. Au travers de l'étude du cas de la certification du Forest Stewardship Council (FSC) à la lumière de la sociologie de la traduction, l'objectif de cet article est de synthétiser les causes de cet échec et de proposer quelques pistes de réflexion afin de tenter d'y remédier.

Mots clefs : certification forestière ; développement durable ; sociologie de la traduction

Codes JEL : Q01 - Développement durable ; Q23 - Sylviculture

\section{THE FAILURE OF FOREST CERTIFICATION}

The FSC certification in the light of the actor-network theory

\begin{abstract}
Initiated in the 1990s, forest certification aims to promote sustainable forest management. However, despite the efforts devoted to its implementation, it does not meet the expected success. Through the study of the Forest Stewardship Council (FSC) certification in the light of the actor-network theory, this article aims to synthesize the causes of this failure and to propose some avenues in order to overcome them.
\end{abstract}

Keywords: actor-network theory; forest certification; sustainable development

JEL Codes: Q01 - Sustainable development ; Q23 - Forestry 
La certification forestière a été créée dans les années 1990 afin de promouvoir des pratiques de gestion forestière durable et de juguler ainsi la déforestation massive que connaissent les pays du Sud ${ }^{1}$ depuis des décennies, jugée en partie responsable de la dégradation de l'environnement et du changement climatique, ainsi que de l'accentuation des inégalités sociales parmi les populations les plus fragiles. À l'heure actuelle cependant, les observateurs s'accordent pour dire que la certification forestière ne rencontre pas le succès escompté.

L'objectif de cet article est de synthétiser les causes de cet échec, lesquelles ont été largement rapportées dans la littérature scientifique mais n'ont pas encore été recensées et articulées au sein d'un cadre analytique cohérent, pour proposer ensuite quelques pistes de réflexion qui pourraient permettre d'y remédier. Pour ce faire, nous étudierons le cas emblématique du développement de la certification Forest Stewardship Council (FSC) à la lumière de la sociologie de la traduction. Au travers de ce travail de synthèse, nous montrons 1) que les questions posées par la certification ont été mal identifiées et 2) que, en conséquence, les moyens mis en œuvre ne permettent pas son application dans les forêts du Sud, en particulier par les petits gestionnaires et exploitants.

Le papier est structuré de la manière suivante. Premièrement, nous dressons le bilan mitigé des deux décennies de certification forestière. Deuxièmement, nous illustrons ce phénomène à partir d'une étude historique de la certification FSC à la lumière de la sociologie de la traduction, en prenant appui sur la littérature scientifique existante ainsi que sur des rapports rédigés par de grands organismes de recherche. Troisièmement, en conclusion, nous proposons quelques préconisations qui pourraient permettre de surmonter l'échec que connaît actuellement la certification forestière.

\section{La certification forestière, un constat d'échec}

Initiée dans les années 1990, la certification forestière a pour objectif de favoriser le recours à des pratiques forestières durables. Elle s'inscrit dans une démarche plus générale de développement de l'écocertification, qui vise à promouvoir la durabilité dans les pratiques productives ainsi que l'insertion des produits certifiés sur le marché mondial.

La certification forestière est délivrée par un organisme indépendant suite à la demande des postulants. Elle résulte généralement de la volonté de ces derniers de bénéficier d'un avantage concurrentiel ou d'éviter un désavantage concurrentiel sur le marché (Gulbrandsen, 2006 ; Overdevest et Rickenbach, 2006). La certification peut concerner l'exploitation forestière elle-même, c'està-dire le premier chaînon de production, ou bien les différents acteurs de la chaîne de contrôle (Chain of Custody - CoC). Le système de certification peut

${ }^{1}$ Dans cet article, le Sud concerne les zones géographiques abritant des forêts tropicales et sub-tropicales, en Amérique du Sud, en Afrique et en Asie. 
être reconnu nationalement (e.g., le Canadian Standards Association - CSA Canada, le Programa Brasileiro de Certificação Florestal - Cerflor Brésil, le Système Panafricain de Certification Forestière au Gabon - PAFC Gabon) ou internationalement (FSC et $\mathrm{PEFC}^{2}$ ), et résulter de l'initiative de gouvernements (e.g., CSA, Cerflor), d'ONG environnementales (FSC) ou de l'industrie du bois (PEFC).

Les forêts certifiées couvrent actuellement 417 millions d'hectares dans le monde, soit $10 \%$ de la surface forestière globale, et leur nombre ne cesse d'augmenter (+ 32,8 millions d'hectares entre 2012 et 2013). Leur rôle économique est considérable. À titre d'exemple, le bois rond industriel (i.e., la matière brute avant transformation) en provenance de forêts certifiées représente environ 28,3\% de l'offre globale (FAO, 2011, 2013). En outre, la certification $\mathrm{CoC}$ est également en plein essor : nous assistons à une augmentation de plus de 8\% de cette certification depuis mars 2013 pour le FSC, soit 27367 certifications (FSC, 2014), et à une augmentation de 4,7\% entre 2012 et 2013 pour le PEFC, soit 9996 certifications (PEFC, 2014).

Pourtant, les constats de disparités entre les forêts situées dans les pays du Nord et celles situées dans les pays du Sud se sont récemment multipliés dans la littérature académique ainsi que dans les rapports des grands organismes de recherche (e.g., Buttoud et Karsenty, 2001 ; Ebeling et Yasué, 2009 ; FAO, 2011 ; Klooster, 2005 ; OIBT, 2010 ; Ozinga, 2004 ; Rametsteiner et Simula, 2003). Ainsi, les forêts tempérées et boréales des pays du Nord concentrent environ 97\% de la certification PEFC (PEFC, 2014) et environ 84\% de la certification FSC (FSC, 2014). De même, le bois rond industriel provient à 95,8\% des forêts européennes et d'Amérique du Nord, laissant peu de place aux produits des forêts du Sud (FAO, 2013). Les forêts tropicales et sub-tropicales des pays du Sud restent donc largement absentes.

De la même manière, les disparités entre les opérations forestières de grande et de petite échelle ont été largement soulignées (e.g., Ebeling et Yasué, 2009 ; Klooster, 2010 ; McDermott, 2012 ; Nussbaum et al., 2000). Les propriétaires ou gestionnaires de petites forêts, notamment familiales ou communautaires, sont en effet concernés de manière marginale par la certification au profit des grandes exploitations industrielles, pour lesquelles les coûts directs ${ }^{3}$ liés à la certification sont relativement bas (Gullison, 2003). En outre, certaines mesures mises en œuvre par le FSC afin de s'adapter aux spécificités des petits gestionnaires forestiers, telles que la certification en groupe ou la certification régionale, semblent entacher sa légitimité et créer autant de problèmes qu'elles semblent en résoudre (Nussbaum et al., 2000).

\footnotetext{
${ }^{2}$ Le PEFC est un label environnemental qui accrédite les schémas nationaux de certification. Il en reconnaît aujourd'hui 34 (PEFC, 2014).

${ }^{3}$ Les coûts directs de la certification sont liés au processus d'obtention de la certification lui-même. Les coûts indirects correspondent aux changements que l'entreprise doit réaliser pour atteindre les standards du système de certification. Bien que les coûts indirects puissent être parfois élevés pour les pays du Nord, ils le sont généralement encore plus pour ceux du Sud (Gullison, 2003).
} 
La littérature avance de nombreuses causes de l'échec de la certification dans les forêts du Sud, et ce, en particulier pour les petits gestionnaires et exploitants : les coûts directs et indirects importants de la certification (Gullison, 2003), l'incertitude foncière et les conflits sur la terre (e.g., Ebeling et Yasué, 2009, pour l'Amérique latine), l'absence d'un prix premium sur les produits certifiés (Guéneau et Tozzi, 2008), le manque d'incitations et de soutien de la part des gouvernements (pour le cas de l'Afrique, voir Buttoud et Karsenty, 2001), etc. Néanmoins, ces différents problèmes n'ont pas été recensés au sein d'un cadre analytique cohérent.

Dans la partie suivante, nous proposons donc de recenser et d'articuler les difficultés rencontrées par la certification forestière. Pour ce faire, nous étudierons le cas emblématique du développement de la certification Forest Stewardship Council (FSC) à la lumière de la sociologie de la traduction. Cette analyse nous permettra ensuite de proposer quelques pistes de réflexion qui pourraient permettre de surmonter l'échec actuel.

\section{Le cas de la certification FSC à la lumière de la sociologie de la traduction}

Afin d'éclairer l'échec rencontré par la certification forestière, nous avons choisi d'étudier le cas de la certification FSC à la lumière de la sociologie de la traduction (voir Tableau). D'une part, le Forest Stewardship Council (FSC) bénéficie d'une large reconnaissance au niveau mondial, ainsi que d'une certaine légitimité. En outre, de nombreuses données sont disponibles dans la littérature scientifique ainsi que dans les rapports rédigés par de grands organismes de recherche (e.g., FAO). Nous les emploierons dans le cadre de cet article.

D'autre part, le cadre théorique de la sociologie de la traduction propose une démarche en quatre étapes, qualifiée de processus de traduction, qui permet de distinguer les différents moments d'un changement et les difficultés qui peuvent être rencontrées (Akrich et al., 2006 ; Callon, 1986). Nous y aurons recours afin de mettre en évidence 1) les différentes étapes du changement dans les pratiques de gestion forestière que constitue le développement de la certification FSC ainsi que 2) les problèmes rencontrés à chacune de ces étapes qui permettent d'expliquer l'échec actuel de la certification forestière.

Tableau. Le processus de traduction de la certification FSC

\begin{tabular}{|l|l|l|l|}
\hline $\begin{array}{l}\text { Étapes du } \\
\text { processus de }\end{array}$ & Dates & $\begin{array}{l}\text { Mise en place de la } \\
\text { certification FSC }\end{array}$ & $\begin{array}{l}\text { Problèmes identifiés lors } \\
\text { de l'étape }\end{array}$ \\
\hline
\end{tabular}




\begin{tabular}{|c|c|c|c|}
\hline traduction & & & \\
\hline $\begin{array}{l}\text { Problématisation. } \\
\text { Ou l'émergence du } \\
\text { principe de la } \\
\text { certification FSC }\end{array}$ & $\begin{array}{l}1992- \\
1993\end{array}$ & $\begin{array}{l}\text { Volonté de mise en } \\
\text { place d'un système } \\
\text { de certification afin } \\
\text { de diffuser des } \\
\text { produits durables } \\
\text { issus des forêts } \\
\text { tropicales sur le } \\
\text { marché mondial. }\end{array}$ & $\begin{array}{l}\text { Mise en avant de la } \\
\text { régulation hors État } \\
\text { (Cashore, 2002) et de } \\
\text { l'écologisme libéral } \\
\text { (Bernstein, 2001). }\end{array}$ \\
\hline $\begin{array}{l}\text { Intéressement. } \\
\text { Ou la mobilisation } \\
\text { des parties } \\
\text { prenantes }\end{array}$ & $\begin{array}{l}1992 \text { - } \\
\text { présent }\end{array}$ & $\begin{array}{l}\text { Concertations entre } \\
\text { représentants des } \\
\text { parties prenantes de } \\
\text { pays du Nord et du } \\
\text { Sud. }\end{array}$ & $\begin{array}{l}\text { Sous-représentation des } \\
\text { intérêts des pays du Sud } \\
\text { (Dingwerth, 2008; Guedes } \\
\text { Pinto et McDermott, 2013) } \\
\text { et absence des } \\
\text { gouvernements lors des } \\
\text { négociations (Cashore et } \\
\text { al., 2004). }\end{array}$ \\
\hline $\begin{array}{l}\text { Enrôlement. } \\
\text { Ou la définition des } \\
\text { modalités de mise } \\
\text { en œuvre de la } \\
\text { certification FSC }\end{array}$ & $\begin{array}{l}1994 \text { - } \\
\text { présent }\end{array}$ & $\begin{array}{l}\text { Adoption par vote des } \\
\text { Principes et Critères } \\
\text { (P\&C) de gestion } \\
\text { durable des forêts, } \\
\text { applicables à tout } \\
\text { type de forêt. }\end{array}$ & $\begin{array}{l}\text { Focalisation de la } \\
\text { durabilité autour de } \\
\text { critères de performance } \\
\text { écologique (Brédif, 2008). }\end{array}$ \\
\hline $\begin{array}{l}\text { Mobilisation } \\
\text { d'alliés. } \\
\text { Ou le } \\
\text { développement du } \\
\text { recours à la } \\
\text { certification FSC }\end{array}$ & $\begin{array}{l}1996 \text { - } \\
\text { présent }\end{array}$ & $\begin{array}{l}\text { Mise en œuvre de la } \\
\text { certification FSC par } \\
\text { les acteurs } \\
\text { concernés. }\end{array}$ & $\begin{array}{l}\text { Des problèmes en matière } \\
\text { de diffusion auprès des } \\
\text { ONG, des gouvernements, } \\
\text { et des consommateurs } \\
\text { (Brédif, } 2008 \text {; Buttoud et } \\
\text { Karsenty, } 2001 \text {; Tozzi et } \\
\text { al., 2011). } \\
\text { Des problèmes également } \\
\text { en matière d'adoption, en } \\
\text { particulier par les pays du } \\
\text { Sud, du fait d'une } \\
\text { certification pas toujours } \\
\text { utile, et d'un transfert de } \\
\text { ressources insuffisant } \\
\text { (Buttoud et Karsenty, } \\
\text { 2001 ; Thornber et al., } \\
1999 \text {; van Kooten et al., }\end{array}$ \\
\hline
\end{tabular}




\subsection{La problématisation, ou l'émergence du principe de la certification FSC}

Appelée problématisation, la première étape d'un processus de traduction consiste à identifier les parties prenantes à un changement ainsi que leurs points de vue respectifs par rapport à celui-ci, ceci afin d'en proposer une formulation générale - une problématisation - qui soit de nature à répondre à leurs préoccupations et à leurs attentes (Akrich et al., 2006 ; Callon, 1986). Il s'agit également de définir la place de chacun des acteurs dans ce changement, ce qui permettra par la suite de souligner l'importance de leur participation à sa mise en œuvre.

Le problème général de la préservation de la biodiversité se pose à partir des années 1970 et 1980. À l'époque, les dégâts écologiques se font si intenses et si évidents (e.g., le réacteur nucléaire de Tchernobyl, la fuite de l'usine de pesticides à Bhopal, la sécheresse en Afrique) que des initiatives voient le jour afin d'endiguer les impacts négatifs de la croissance économique et de l'industrie sur la nature. D'une part, la communauté scientifique commence à s'intéresser de près aux problématiques écologiques liées à l'activité économique (e.g., Dasgupta et Heal, 1979 ; Georgescu-Roegen, 1971 ; Passet, 1979). D'autre part, les activistes et les organisations environnementales des pays du Nord s'emparent également de ces enjeux en sensibilisant le public, notamment autour de la conservation de la biodiversité, qui est alors largement associée aux forêts tropicales (Boisvert et Vivien, 2012).

En ce qui concerne le problème plus particulier de la préservation des forêts tropicales, l'Accord International sur les Bois Tropicaux (AIBT) est signé en 1983 (Humphreys, 1996). Cependant, la réglementation n'est pas acceptée car elle n'est pas compatible avec les règles du commerce du GATT ${ }^{4}$, qui prônent un commerce libre et une régulation décentralisée sans intervention des États (Bernstein et Cashore, 2004 ; Mersmann, 2004). L'AIBT n'étant pas jugé satisfaisant, de nouvelles discussions prennent place dans le but de présenter une Convention sur la forêt lors du Sommet de la Terre à Rio de Janeiro en 1992. La tentative de Convention est un nouvel échec. Les parties ne parviennent à aucun accord sur les responsabilités de chacun quant à la gestion des forêts, le débat opposant fermement les pays du Nord et ceux du Sud (Bernstein et Cashore, 2004 ; Brédif, 2008).

Suite à cet échec, un groupe de réflexion mené par la World Wide Foundation (WWF) est créé autour de la question du développement durable et de la gestion des forêts. Le WWF est l'acteur moteur du changement - le traducteur au sens de la sociologie de la traduction -, puisqu'il initie et coordonne les négociations

${ }^{4}$ Le GATT est le General Agreement on Tariffs and Trade qui deviendra en 1994 l'Organisation Mondiale du Commerce (OMC). 
entre les différents acteurs composant ce groupe hétérogène d'individus et d'organisations : Organisations Non Gouvernementales (ONG) environnementales, utilisateurs du bois et entreprises forestières. Certaines antennes nationales de WWF avaient déjà été instigatrices du mouvement de boycott des bois tropicaux au milieu des années 1980, conjointement avec d'autres ONG environnementales, ce qui témoigne de l'engagement précoce du WWF dans la question forestière. Au moment de la création du FSC, l'importance du WWF International est donc considérable, ce qui lui permet de recevoir le soutien de la Banque mondiale pour la promotion de la gestion durable des forêts tropicales (Buttoud et Karsenty, 2001).

Afin de faire face à l'échec des négociations antérieures, et pour s'inscrire dans la lignée d'un projet de développement durable ${ }^{5}$ avantageant le pilier économique au détriment des deux autres piliers (environnemental et social), où le marché représente la figure centrale de la coordination économique, et où il est possible d'internaliser la contrainte environnementale sans remettre en cause le paradigme économique néoclassique dominant (Figuière, 2006), la proposition apportée par ce groupe de réflexion s'inspire alors de recommandations préconisées par le Consensus de Washington appliquées à la question environnementale. Il s'agit de mettre en place une forme d'écologisme libéral (« liberal environmentalism », Bernstein, 2001), dans lequel la régulation décentralisée de la gestion des ressources naturelles - ici, les forêts -, le commerce international et une moindre implication des gouvernements sont des éléments centraux (Damian et Graz, 2001).

Un type différent de gouvernance émerge alors, qui détient son autorité non pas de l'État, mais des marchés mondiaux et de la prise en compte des préférences des consommateurs ${ }^{6}$ (Cashore, 2002). Cette nouvelle forme de gouvernance est particulièrement bien illustrée par la certification forestière et par le FSC (Gulbrandsen, 2004). Les ONG environnementales, dont le WWF et Greenpeace, travaillent main dans la main avec le marché - mais sans discussions avec les gouvernements -, lequel n'est alors plus considéré comme le problème mais comme la solution à la dégradation des forêts tropicales (Boisvert et Vivien, 2012 ; Brédif, 2008). Le principe de la certification forestière a ainsi été défini en tant que mécanisme incitatif de marché qui oblige les entreprises à internaliser les coûts de la gestion durable de la forêt afin de décrocher la certification et d'obtenir en conséquence un prix premium sur le marché du bois. La certification forestière est censée garantir de la sorte l'approvisionnement du marché mondial de produits ligneux issus de forêts tropicales gérées durablement, satisfaisant ainsi une demande émanant des

\footnotetext{
${ }^{5}$ La notion de développement durable est hautement polysémique, nous ne reviendrons pas sur ce débat. Ce qui nous intéresse ici est de noter que la « version Brundtland » du développement durable s'est imposée à partir des années 1980-1990 (Figuière, 2006). C'est cette version du développement durable à laquelle nous nous référons.

${ }^{6}$ En principe, c'est le consommateur qui régule les flux de bois tropicaux en choisissant d'acheter ou non des produits certifiés. La certification offre ainsi une alternative au boycott des bois tropicaux prôné au milieu des années 1980 par des ONG environnementales, dont WWF et Greenpeace. Il est intéressant de noter que ce sont les consommateurs du Nord (Europe de l'Ouest et Amérique du Nord) qui sont les plus sensibles d'abord au boycott, puis à l'achat de produits écocertifiés (Buttoud et Karsenty, 2001 ; FAO, 2006, 2013).
} 
consommateurs. Elle est l'illustration archétypale de la mise en œuvre d'un écologisme libéral au travers d'un système de gouvernance hors État (Brédif, 2008 ; Gulbrandsen, 2006).

\subsection{L'intéressement, ou la mobilisation des parties prenantes}

À l'issue de l'étape de problématisation, il s'agit ensuite d'associer les acteurs au changement au travers d'une seconde phase, qualifiée d'étape d'intéressement. Celle-ci a pour objectif de faire venir l'ensemble des parties prenantes préalablement identifiées à la table des négociations, en tentant de les convaincre de l'intérêt de la problématique soulevée (Akrich et al., 2006 ; Callon, 1986).

Dans cette perspective, un groupe d'acteurs hétérogènes, futurs membres du FSC, composé d'utilisateurs de produits ligneux, d'ONG environnementales et d'entreprises de la filière bois, se réunit en 1990 en Californie afin de discuter de l'avenir de la gestion forestière dans les forêts tropicales. En mars 1992, un conseil d'administration provisoire du FSC est établi à Washington. Dès l'année suivante, les premières certifications sont délivrées au Mexique et aux ÉtatsUnis. L'attention est alors portée sur la nécessité d'accroître la représentation des intérêts des pays du Sud ainsi que celle des peuples indigènes (Dingwerth, 2008). En octobre 1993, la première assemblée générale regroupant quelques 130 participants de 26 pays a lieu au Canada, à Toronto, institutionnalisant ainsi le FSC. L'année suivante, le premier secrétariat du FSC s'établit à Oaxaca au Mexique. Il sera déplacé à Bonn en Allemagne en 2003.

Malgré une bonne volonté manifeste, deux problèmes apparaissent d'emblée en matière de mobilisation des parties prenantes. Le premier concerne la nature des acteurs mobilisés. Le FSC est en effet fondé par un partenariat entre des acteurs privés et la société civile, excluant les gouvernements de la négociation. Le FSC est ainsi parfois réputé être «le cas le plus avancé d'établissement de règles non impulsées par l'État dans le domaine de l'environnement » (Gulbrandsen, 2004, 76, traduction des auteurs).

Pourtant, en matière de gouvernance, l'État n'est pas un acteur neutre, étant au contraire au cœur de la régulation environnementale (Brand et Görg, 2003). Les gouvernements sont en outre réputés avoir un rôle significatif dans le succès des mécanismes de régulation privés, et donc dans la promotion de la certification forestière (Cashore et al., 2004). Ils peuvent par exemple mettre en place des politiques et des réglementations favorisant l'importation de produits ligneux certifiés (Gulbrandsen, 2006), agir par le biais de leur consommation de bois certifié pour la construction civile et les projets d'équipements (Mersmann, 2004), verser des aides pour le fonctionnement du FSC (Tozzi et al., 2011), ou encore, soutenir directement la création des institutions de certification dans des pays en développement (e.g., l'Agence française de développement et ses actions en Afrique centrale). Leur place est donc tout à fait importante, tant 
dans leur rôle classique de gouvernance - en contribuant par eux-mêmes à la gestion durable des forêts -, comme dans leur rôle de méta-gouvernance - en participant à la création d'un système de gouvernance des forêts (VisserenHamakers et Glasbergen, 2007). Enfin, nombre d'auteurs qui ont travaillé sur la gouvernance mondiale s'accordent à dire que l'absence des gouvernements des négociations implique un manque de souveraineté dans l'élaboration des critères et des priorités établies dans les mécanismes de gouvernance - ici le FSC - ce qui en réduit la légitimité (Cashore et al., 2004).

Le second problème qui apparaît au cours de cette phase d'intéressement porte sur la mobilisation effective des acteurs. En théorie, la gouvernance du FSC est censée assurer l'équitable représentation des intérêts du Nord et du Sud ainsi que celle des trois piliers du développement durable - social, économique et environnemental - au sein de son processus de décision (voir Schéma). Pour ce faire, la gouvernance du FSC est organisée autour de trois chambres, chacune affectée à l'un des trois piliers du développement durable, qui se réunissent en Assemblée Générale (AG), ainsi que d'un Conseil d'Administration (CA) et d'une équipe exécutive. Les membres des trois chambres - sociale, économique et environnementale -, individus ou organisations, possèdent tous un droit de vote, et le poids des représentants du Sud et du Nord est identique quel que soit le nombre de participants ${ }^{7}$.

En pratique cependant, l'intéressement des parties prenantes semble établir une relation de supériorité en faveur du Nord. Premièrement, les activistes des pays du Sud déplorent le fait que les intérêts des parties prenantes du Nord sont davantage pris en compte et que, partant, les forêts boréales et tempérées sont davantage ciblées par la certification. Deuxièmement, la place des pays du Sud dans la prise de décision n'est pas aussi évidente qu'il n’y paraît. Les pays hébergeant les forêts du Sud regroupent 75\% de la population, mais ne représentent que $50 \%$ des votes des trois chambres. Le poids relatif de chaque membre du Sud est donc inférieur à celui d'un membre du Nord ${ }^{8}$. Enfin, troisièmement, le même type de constat peut être fait quant à la distribution des membres au sein des chambres. En 2011, ce sont les chambres environnementale et économique qui comprenaient le plus de membres (respectivement $45 \%$ et $36 \%$ ). Il semble donc que la chambre sociale soit largement sous-représentée et inadaptée aux ambitions du FSC qui était, rappelons-le, d'accorder une importance toute aussi grande aux trois chambres (Guedes Pinto et McDermott, 2013).

\section{Schéma. Organigramme du FSC (à partir de www.fsc.org)}

\footnotetext{
${ }^{7}$ Les décisions de l'AG doivent être adoptées par au moins les 2/3 des voix. Le CA est élu par les membres du FSC. L'équipe exécutive se charge quant à elle de la gestion au jour le jour du FSC. Elle a notamment un rôle important dans l'interprétation des P\&C (Dingwerth, 2008).

${ }^{8}$ En outre, la distribution des sièges occupés par le Sud et le Nord au CA révèle des disparités pour le Nord comme pour le Sud (sur-représentation de l'Europe et de l'Amérique Latine : 7 sièges sur 9 disponibles).
} 


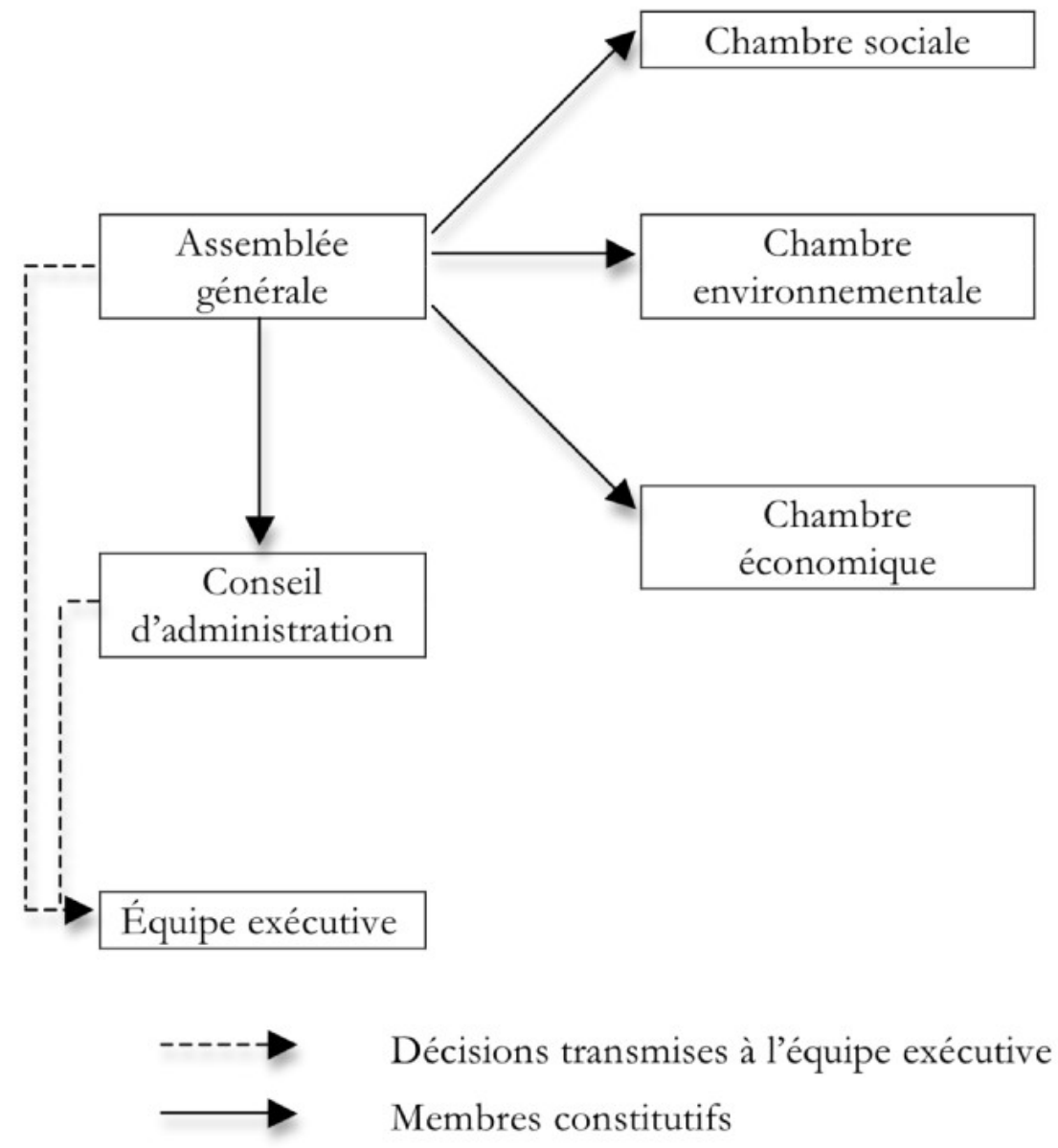

\subsection{L'enrôlement, ou la définition des modalités de mise en oeuvre de la certification FSC}

La troisième étape d'un processus de traduction est celle de l'enrôlement. Celleci consiste à définir les modalités concrètes de mise en œuvre du changement et ce, en concertation avec les acteurs (Akrich et al., 2006 ; Callon, 1986).

En l'occurrence, les fondateurs du FSC ont approuvé en 1994 neuf Principes qui servent de règles à la gestion durable des forêts, subdivisés en Critères qui fournissent les moyens d'évaluer le respect de chacun de ces Principes ${ }^{9}$. À portée normative, ces Principes et Critères $(P \& C)$ servent aujourd'hui encore de guide au FSC ainsi qu'aux organismes d'évaluation qui délivrent la certification (FSC, 2012). Ces P\&C sont basés sur la notion de performance et axés sur les résultats (Tozzi et al., 2011). Cette orientation est d'ailleurs explicitement

\footnotetext{
${ }^{9}$ Depuis 1996, il y a dix Principes. Le nombre de Critères a également augmenté périodiquement. Dans la version la plus récente des P\&C (2012), il y en a 70. Ils sont aisément accessibles sur le site du FSC, à l'adresse : http://fr.fsc.org/principeset-critres-fsc. 278.htm
} 
énoncée dès le préambule des Principes et Critères de Gestion forestière FSC : «Le FSC a publié pour la première fois les Principes et Critères FSC [...] comme un standard international, basé sur les performances et orientés vers les résultats. Les Principes et Critères s'intéressent à la performance sur le terrain de la gestion forestière plutôt qu'aux systèmes de gestion pour atteindre cette performance sur le terrain. » (FSC, 2012, 7, emphase des auteurs).

En outre, ces $P \& C$ ont vocation à servir de standard mondial applicable à toutes les forêts - et pas seulement aux forêts tropicales - quels que soient leur position géographique, leur taille ou leur type de végétation (FSC, 2012). Les $\mathrm{P} \& \mathrm{C}$ sont par ailleurs complémentaires des lois et des réglementations locales, nationales et internationales (Principe $1 \mathrm{du}$ FSC). Afin de faciliter la mise en place des P\&C au sein des nations, le FSC dispose de bureaux nationaux dans 40 pays, notamment en Europe et en Amérique latine, ainsi que de quatre bureaux régionaux (en Europe, en Amérique latine, en Russie, en Afrique, et en Asie).

La version actuelle des P\&C a été adoptée à 75\% des voix en 2012, clôturant ainsi un processus consultatif de trois ans. Il s'agit de la cinquième version des P\&C depuis leur création en 1994, la prochaine révision devant débuter en 2015. Les $\mathrm{P} \& \mathrm{C}$ sont établis de sorte à être en conformité avec trois principaux documents de portée internationale : en matière de légitimité des certifications, avec l'International Social and Environmental Accreditation and Labelling (ISEAL), en matière de normalisation, avec l'Organisation Internationale de Normalisation/Commission Électronique Internationale (ISO/IEC), ainsi qu'en matière de réglementation du commerce international, avec l'Accord de l'OMC sur les Obstacles Techniques au Commerce (OTC).

Bien que le FSC revendique l'évaluation d'une gestion durable de la forêt au travers de ses $\mathrm{P} \& \mathrm{C}$, les travaux montrent que ces derniers se contentent en réalité de décrire l'état écologique des forêts et de certaines fonctions remplies par celles-ci (Brédif, 2008 ; Guedes Pinto et McDermott, 2013) : « En insistant sur l'importance des niveaux de performance à atteindre à l'échelle de l'unité de gestion forestière à certifier [...], [le FSC] parachève sans complexe la logique substantielle de la durabilité. » (Brédif, 2008, 17).

Dans le cadre de l'obtention de la certification FSC, l'organisme réalisant l'évaluation d'une entreprise établit une liste d'éléments à vérifier sur la base des Critères. Le FSC, qui fournit la liste des Critères, cherche à laisser une place la plus infime possible à l'interprétation, tant pour simplifier l'évaluation que pour gagner en crédibilité. Les Critères ont ainsi une portée très technique se rapportant essentiellement à la performance écologique des forêts. À titre d'exemple, le nombre autorisé d'arbres à abattre est spécifié selon l'espèce, la valeur commerciale, et ce, pour chaque hectare de parcelle de forêt (FSC, 2012).

Faisant écho à la diffusion des idées et des valeurs du développement durable - 
dans sa version Brundtland - et de l'écologisme libéral déjà évoquées précédemment (Bernstein, 2001), la gestion durable des forêts s'est ainsi progressivement écartée de références à des valeurs sociales (Brédif, 2008). Au sein du FSC, les fonctions écologiques de la forêt sont mises en avant au détriment de ses autres fonctions, comme par exemple celles culturelles ou sociales. La durabilité à laquelle font référence les P\&C du FSC se focalise en effet sur la performance écologique, qui permet d'assurer la viabilité économique du secteur et d'approvisionner le marché mondial en produits ligneux issus de forêts gérées durablement.

\subsection{La mobilisation d'alliés, ou le développement du recours à la certification FSC}

Enfin, la quatrième et dernière étape d'un processus de traduction est celle de la mobilisation d'alliés. La négociation du changement se déroulant bien souvent dans un cercle restreint - via des porte-paroles -, il s'agit finalement de propager ce changement auprès de l'ensemble des acteurs concernés afin qu'ils l'adoptent (Akrich et al., 2006 ; Callon, 1986).

Ainsi, une fois débattus, les P\&C du FSC doivent ensuite être diffusés auprès des acteurs du secteur qui seront susceptibles de les adopter. Dans cette perspective, la légitimité de la certification FSC doit préalablement être établie afin que celle-ci apparaisse comme étant un système fiable d'évaluation de la durabilité environnementale, économique et sociale. Pour ce faire, le système de certification doit être transparent, garantir des performances minimales, être cohérent et reproductible, et il faut que les parties prenantes y soient associées (Ozinga, 2004). En l'occurrence, le FSC ne semble pas rencontrer de problèmes particuliers à ce niveau.

Une fois la légitimité établie, il est ensuite nécessaire de diffuser les modalités de certification auprès des acteurs de la filière bois (du propriétaire, gestionnaire ou exploitant forestier au revendeur détaillant), des consommateurs, des gouvernements (qui ont un rôle de régulateur), ainsi que de la société civile. À ce titre, les ONG environnementales sont des acteurs très importants dans la transmission de normes écologiques et sociales. Le FSC bénéficie de l'appui de très nombreuses ONG reconnues sur la scène mondiale, telles que WWF et Greenpeace. Cependant, il faut noter que certaines ONG telles que la Rainforest Foundation ne sont pas favorables au FSC (Brédif, 2008 ; Buttoud et Karsenty, 2001 ; Tozzi et al., 2011). De même, pour certains pays la présence d'ONG est gênante dans leur propre gouvernance des forêts (Buttoud et Karsenty, 2001). Il convient aussi de noter les difficultés auxquelles doivent faire face les ONG pour traduire un discours sur la durabilité dans des contextes nationaux et régionaux hétérogènes. Enfin, les consommateurs ne sont pas toujours informés de l'existence de produits forestiers certifiés.

Par delà ces problématiques en matière de diffusion, la certification FSC 
rencontre également toute une série de difficultés en matière d'adoption. Deux grands ensembles de facteurs font obstacle au développement de la certification FSC. Premièrement, la certification FSC ne semble pas pertinente pour nombre d'acteurs de la filière, tels que certains propriétaires, gestionnaires ou exploitants. D'une part, le marché des produits ligneux certifiés étant émergent, il est nécessaire de stimuler la demande qui lui est adressée ${ }^{10}$. Dans cette perspective, le WWF a participé à développer le marché des produits certifiés, en créant, dès 1991, un groupe d'achat, le Global Forest \& Trade Network (GFTN), qui regroupe plus de 800 entreprises dans une trentaine de pays (Klooster, 2005). Cependant, que ce soit en raison des problèmes d'information précédemment évoqués, ou par manque d'intérêt ou de pouvoir d'achat, les efforts du côté de la demande ne sont pas à la hauteur de l'augmentation de l'offre de produits certifiés (Katila et Puustjärvi, 2004). D'autre part, les acteurs les plus susceptibles d'avoir recours à la certification sont ceux qui ont une activité d'exportation, notamment vers les pays du Nord dont les consommateurs sont plus sensibles à l'écocertification (Gulbrandsen, 2006). Or, ces dernières décennies, le commerce Sud-Sud se développe, ce qui ne favorise en rien la diffusion du FSC. Nombre de pays du Sud, comme le Brésil, emploient la production issue de leurs propres forêts presque entièrement sur leur marché domestique - 80\% de la production dans le cas brésilien (ABIMCI, 2009) -, moins exigeant en matière de certification.

Deuxièmement, quand bien même ils souhaiteraient l'adopter, nombre d'acteurs du secteur forestier ne peuvent accéder à la certification FSC, dans la mesure où le FSC n'assure pas de transfert de ressources nécessaires à son obtention (Thornber et al., 1999 ; van Kooten et al., 2005). L'important coût financier et technique de la certification est subi en amont de la filière, par le propriétaire, le gestionnaire ou l'exploitant, sur simple pression de la chaîne de production et des ONG. Il n'est pas excessif de penser que les petites forêts du Sud sont celles qui ont le plus de modifications et d'aménagements à apporter pour atteindre les critères du FSC (Ebeling et Yasué, 2009), ce qui entraîne des coûts indirects liés à la mise en place de la certification pouvant être très élevés, en particulier dans le cas d'exploitations ayant lieu dans des pays où la réglementation forestière est faible ou peu contrôlée ${ }^{11}$ (Gullison, 2003). Ces dernières ont alors un coût total de certification plus élevé, ce qui en réduit considérablement la rentabilité. Il est dès lors difficile d'imaginer que le petit propriétaire cherchera à mettre en place des pratiques non rentables de gestion forestière durable (Karsenty, 2002).

\section{Conclusion : quelles pistes pour surmonter l'échec actuel ?}

\footnotetext{
${ }^{10}$ Nous n'étudions pas ici l'effet prix que cela pourrait entraîner sur les choix de consommation, dans la mesure où un prix premium n'est pas systématiquement constaté sur les produits certifiés (FAO, 2011 ; Guéneau et Tozzi, 2008).

${ }^{11}$ Gullison évoque ainsi le cas d'une entreprise brésilienne dont les coûts d'exploitation avec la certification ont augmenté de $30 \%$ par rapport à ses anciennes pratiques forestières conventionnelles (Gullison, 2003).
} 
Ces dernières années, de nombreuses raisons ont été avancées afin d'expliquer l'échec rencontré par la certification forestière, sans pour autant en offrir une explication globale. Notre analyse historique du déploiement de la certification FSC à la lumière de la sociologie de la traduction nous a permis de recenser et d'articuler ces divers éléments au sein d'un cadre analytique cohérent. Ce cadre nous permet ainsi de mettre clairement en évidence le problème de fond que rencontre la mise en œuvre de la certification forestière depuis ses débuts, à savoir, celui de la régulation de la gestion forestière par le marché, qui se traduit par des modalités d'application tournées vers une vision restreinte en termes de durabilité. En conséquence, telle qu'elle est actuellement mise en œuvre, la certification forestière ne permet pas de répondre aux préoccupations environnementales et sociales relatives aux forêts du Sud, en particulier par les petits gestionnaires et exploitants.

Pour surmonter ce problème, le FSC a amendé ses outils afin de créer des procédures spécifiques aux petits gestionnaires et exploitants du Sud - c'est-àdire aux gestionnaires et exploitants de petites forêts, de forêts familiales ou de forêts communautaires - en matière de formation, de commercialisation, et de financement (FSC, 2012). Mais ces efforts du FSC (e.g., les procédures Small or Low-Intensity Managed Forest - SLIMF - destinées à adapter les normes FSC aux petits propriétaires) ne suffisent pas à améliorer les ventes et à dépasser les barrières imposées par les spécificités territoriales, que l'on considère la production communautaire ou indigène, les exploitations de faible intensité, les tensions sur le statut de la terre, ou encore, le partage des bénéfices avec les populations locales (Buttoud et Karsenty, 2001 ; Guedes Pinto et McDermott, 2013).

Alors, que faudrait-il faire ? Puisque des problèmes ont été rencontrés à chacune des étapes du processus de traduction, la réponse est simple : il faudrait revoir l'ensemble du processus. Ainsi, premièrement, en matière de problématisation, il conviendrait de redéfinir le principe de la certification FSC dans une approche élargie de la durabilité, qui placerait les critères sociaux comme l'objectif premier. Deuxièmement, en matière d'intéressement, il serait pertinent de mobiliser davantage les pays du Sud et d'impliquer les gouvernements. C'est ainsi qu'il est actuellement envisagé d'avoir recours à d'autres mécanismes réglementaires de préservation de la forêt, privés et publics, comme le dispositif FLEGT (Forest Law Inforcement, Governance and Trade) mis en œuvre par l'Europe et entré en vigueur en 2013, qui replace les États au cœur de la gestion durable des forêts mondiales (e.g., par des politiques d'achat public, par une révision de la législation européenne et de celle des États membres, etc.). Troisièmement, en ce qui concerne l'enrôlement, il s'agirait d'intégrer davantage de critères sociaux dans les modalités concrètes de mise en œuvre de la certification. Enfin, quatrièmement, en ce qui concerne la mobilisation d'alliés, il faudrait mettre les ressources financières et 
techniques à disposition des acteurs de la filière bois des pays du Sud. 


\section{Bibliographie}

ABIMCI (2009) Estudo setorial 2009 ano base 2008, Curitiba, 48 p.

AKRICH M., CALLON M., LATOUR B. (2006) Sociologie de la traduction. Textes fondateurs. Paris, Presses des Mines de Paris, $303 \mathrm{p}$.

BERNSTEIN S. (2001) The Compromise of Liberal Environmentalism, New York : Columbia University Press,

BERNSTEIN S., CASHORE B. (2004) Nonstate global governance: Is forest certification a legitimate alternative to a global forest convention? in Hard choices, soft law: Voluntary standards in global trade, environment and social governance, Kirton J.J., Trebilcock M.J., chapitre 2, Aldershot : Ashgate.

BOISVERT V., VIVIEN F.-D. (2012) Towards a political economy approach to the Convention on Biological Diversity, Cambridge Journal of Economics, 36, n5, 1163-79.

BUTTOUD G., KARSENTY A. (2001) L'écocertification de la gestion des forêts tropicales, Revue Forestière Française, 53, nº6, 691-706.

BRAND U., GÖRG C. (2003) The State and the Regulation of Biodiversity, Geoforum, 34, $\mathrm{n}^{\circ} 2,221-233$.

BRÉDIF H. (2008) Référentiels de durabilité forestière : l'universalité en question, Natures Sciences Sociétés, 16, n³, 209-219.

CALLON M. (1986) Éléments pour une sociologie de la traduction. La domestication des coquilles Saint-Jacques et des marins-pêcheurs dans la baie de Saint-Brieuc, L'Année Sociologique, 36, 169-208.

CASHORE B. (2002) Legitimacy and the privatization of environmental governance: How Non-State Market-Driven (NSMD) governance systems gain rule-making authority, Governance: an international journal of policy, administration and institutions, 15, $n^{\circ} 4,503-529$.

CASHORE B., AULD G., NEWSOM D. (2004) Governing through markets : Forest certification and the emergence of non-state authority, London : Yale University Press.

DASGUPTA P.S., HEAL G.M. (1979) Economic theory and exhaustible resources, Cambridge : Press syndicate of the university.

DAMIAN M., GRAZ J.-C. (2001) Les grands paradigmes, in Damian M., Graz J.-C. Commerce international et développement soutenable, Paris : Economica, 19-55.

DINGWERTH K. (2008) North-South Parity in Global Governance: The Affirmative Procedures of the Forest Stewardship Council, Global Governance, 14, 53-71.

EBELING J., YASUÉ M. (2009) The effectiveness of market-based conservation in the tropics: Forest certification in Ecuador and Bolivia, Journal of Environmental Management, 90, 1145-1153.

FAO (2013) Forest products annual market review 2012-2013, Genève, 155 p.

FAO (2011) Forest products annual market review 2010-2011, Genève, 174 p. 
FAO (2006) Forest Products Annual Market Review, 2005-2006. Genève, 163 p.

FIGUIÈRE C. (2006) Sud, développement et développement durable : l'apport de l'économie politique, VertigO, 7, n², 17p.

FSC (2014) Global FSC certificates : type and distribution, 18 p.

FSC (2012) Principes et Critères de Gestion forestière, 36 p.

GEORGESCU-ROEGEN N. (1971) The entropy law and the economic process. Cambridge : Harvard University Press.

GUEDES PINTO L.F., MCDERMOTT C. (2013) Equity and forest certification : A case study in Brazil, Forest Policy and economics, 30, 23-29.

GUÉNEAU S., TOZZI P. (2008) Towards the privatization of global forest governance?, International Forestry Review, 10, n³, 550-562.

GULBRANDSEN L.H. (2006) Creating markets for eco-labelling: Are consumers insignificant?, International Journal of Consumer Studies, 30, n5, 477-489.

GULBRANDSEN L.H. (2004) Overlapping public and private governance: can forest certification fill the gaps in the global forest regime?, Global Environmental Politics, 4, n², 75-99.

GULLISON R.E. (2003) Does forest certification conserve biodiversity?, Oryx, 37, $\mathrm{n}^{\circ} 2$, 153-165.

HUMPHREYS D. (1996) The Global Politics of Forest Conservation since the UNCED. Environmental Politics, 5, 231-256.

KARSENTY A. (2002) Le rôle controversé de la fiscalité forestière dans la gestion des forêts tropicales : Létat du débat et les perspectives en Afrique centrale, Cahiers d'économie et sociologie rurales, 64.

KATILA M., PUUSTJÄRVI E. (2004) Des marchés pour les services forestiers environnementaux : Réalité et potentiel, Unasylva - FAO, 219, n55, 53-58.

KLOOSTER D. (2010) Standardizing sustainable development? The Forest Stewardship Council's plantation policy review process as neoliberal environmental governance, Geoforum, 41, 117-129.

KLOOSTER D. (2005) Environmental certification of forests: The evolution of environmental governance in a commodity network, Journal of rural studies, 21, 403-417.

MCDERMOTT C.L. (2012) Trust, legitimacy and power in forest certification: A case study of the FSC in British Columbia, Geoforum, 43, 634-644.

MERSMANN C. (2004) Les liens entre le commerce et la gestion durable des forêts : un aperçu, Unasylva - FAO, 219, $\mathrm{n}^{\circ} 55,3-9$.

NUSSBAUM R., GARFORTH M., SCRASE H., WENBAN-SMITH M. (2000) An analysis of current FSC accreditation, certification and standard setting procedures identifying elements which create constraints for small forest owners. Proforest, 123.

OIBT (2010) Annual review and assessment of the world timber situation, Yokohama, 200 p. 
OVERDEVEST C., RICKENBACH M.G. (2006) Forest certification and institutional governance: An empirical study of forest stewardship council certificate holders in the United States, Forest Policy and Economics, 9, 93-102.

OZINGA S. (2004) Les effets de la certification sur la gestion forestière durable : L'heure de la vérification, Unasylva - FAO, 219, n55, 33-38.

PASSET R. (1979) L'économique et le vivant. Paris : Payot.

PEFC (2014) PEFC Global Statistics: SFM \& CoC Certification, 16 p.

RAMETSTEINER E., SIMULA M. (2003) Forest certification: An instrument to promote sustainable forest management?, Journal of Environmental Management, 67, n 87-98.

THORNBER K.D., PLOUVIER D., BASS S. (1999) Certification: Barriers to benefits. A discussion of equity implications. European Forest Institute, Discussion paper 8, Joensuu, Finlande, $46 \mathrm{p}$.

TOZZI P., GUÉNEAU S., NDIAYE A. (2011) Gouverner par les normes environnementales : Jeux d'acteurs et de puissance dans la certification forestière, Espaces et Sociétés, 146, n³, 123-139.

VISSEREN-HAMAKERS I.J., GLASBERGEN P. (2007) Partnerships in forest governance, Global Environmental Change, 17, 408-419.

VAN KOOTEN G.C., NELSON H.W., VERTINSKY I. (2005) Certification of sustainable forest management practices: a global perspective on why countries certify. Forest Policy and Economics, 7, 857-867. 